\title{
Enhanced Continuous Higher Order Sliding Mode Control with Adaptation
}

\author{
C. Edwards*, Y. Shtessel \\ Structures \& Dynamics Research Group, CEMPS, University of Exeter, Exeter, UK \\ Department of Electrical Engineering, University of Alabama, Huntsville, AL, USA
}

\begin{abstract}
This paper proposes a new Continuous Adaptive HOSM control algorithm. The key advantage of the adaption scheme is that it does not require knowledge of the bounds on the matched uncertainty, and the gains themselves are not conservatively overestimated by the adaption scheme - which helps mitigate the problem of chattering. Compared with earlier work, two variable parameters are allowed to adapt and this facilitates much better self-tuning capabilities and improved closed-loop performance.
\end{abstract}

Keywords: Sliding modes, Higher order sliding modes, adaptive control

\section{Introduction}

The most attractive feature of conventional sliding mode control, namely its unique robustness properties, has, at least traditionally, come at the cost of using a high frequency switching control law. This is quite acceptable in many practical systems: e.g. DC/DC converter control and impulsive control of space vehicles [5, 14]. However, in many systems the digital implementation of these control laws, combined with the effects of unavoidable unmodelled parasitic dynamics, leads to low frequency oscillations in the sliding variable known as chattering. This oscillation is subsequently transmitted to the state variable during sliding - which is highly undesirable [4]. Over the last two decades significant progress has been made in terms of the creation of

Email addresses: c.edwards@exeter.ac.uk (C. Edwards*), shtessel@eng.uah. edu (Y. Shtessel) 
so-called higher-order sliding mode (HOSM) control schemes which seek to alleviate the phenomenon of chattering - often by artificially inflating the relative degree. However these schemes still inherently employ discontinuous injection terms - usually 'hidden' and buried within the controller dynamics. Whilst these endeavors have resulted in the formulation of continuous sliding mode controllers - such as the popular super-twisting controller - it has been subsequently shown that, rather than totally eliminating chattering, these controllers only mitigate chattering [4]. Even modern higher order controllers such as those proposed in $[11,12]$, require upper bounds on the disturbances/uncertainty (or the derivatives thereof). In general these bounds are difficult to estimate and these supremum values are embedded in the controller gains. The conservatism resulting from using worst case upper bounds on the disturbances/uncertainty within the controller, compounds the chattering associated with the implementation. This phenomenon has (partly) motivated research in what broadly could be described as 'adaptive sliding mode control'. In this paradigm the gains in the controller, which depend on bounds on the uncertainty, are allowed to adapt. For this to be an effective tool to counteract chattering, the adaptive terms must remain as small as possible, and yet be sufficiently large to guarantee the sliding motion is achieved and maintained $([10,13,2])$. Despite the interest, and the growing literature addressing the problem, finding rigorous and yet effective ways of lowering unnecessarily large gains once sliding is achieved, has proved challenging. One common approach is to include 'leakage' terms - but this often leads to only pseudo-sliding or real-sliding [1]. The problem of adventitiously reducing the gains during sliding was specifically studied in [6], where the continuous finite-time control law from [3] in conjunction with an adaptive supertwisting structure was employed. The disadvantage of the approach in [6] is that only one of the super-twisting parameters was adapted. The other tuning parameter was assumed to be sufficiently large - a feature that may adversely effect chattering. To directly address this problem, this paper employs the continuous finite time control law from [3] in conjunction with a new adaptive super-twisting structure from [7] which adjusts both gains. This will be shown to facilitate much better self-tuning capabilities and improved closed-loop performance. 


\section{Problem Statement}

Consider the generic case ${ }^{1}$ when the (scalar) sliding variable, $\sigma$, to be nullified satisfies

$$
\sigma^{(n)}(t)=u(t)+d(t)
$$

where the integer $n>2$. In (1), $u(t)$ represents the scalar control variable, and $d(t)$ is an unknown disturbance. It is assumed the disturbance $d(t)$ is twice differentiable and its first and second derivatives are bounded, but these bounds are not known. The objective is to compute a continuous HOSM control law to force $\sigma, \dot{\sigma}, \ldots, \sigma^{(n-1)}=0$ in finite time despite the uncertainty represented by the unknown signal $d(t)$ and without knowledge of the bounds on $|\dot{d}(t)|$ and $|\ddot{d}(t)|$. To address this problem an adaptive HOSM controller is proposed in which the gains of the adaptive terms evolve in such a way that they are 'as small as possible', and yet can guarantee sliding is maintained. To achieve this, the scheme will rely on exploiting information contained in the so-called equivalent injection signal $[18,6]$. The main contribution of this paper is in proposing a Continuous Adaptive HOSM control algorithm in which both variable parameters adapt (and are not overestimated). This allows better self-tuning capabilities compared to the case in where only one parameter is adapted [6].

\section{Preliminaries}

The control scheme to address the problem posed in Section 2 will be built from two elements: the continuous finite time controller proposed by [3] in conjunction with a recently proposed fully adaptive super-twisting structure from [7]. The existing results which are built upon in this paper will be summarised in this section in the form two propositions:

Proposition 1: (Bhat \& Bernstein) Consider the system

$$
\sigma^{(n)}=\gamma_{1}|\sigma|^{\alpha_{1}} \operatorname{sign}(\sigma) \ldots+\gamma_{n}\left|\sigma^{(n-1)}\right|^{\alpha_{n}} \operatorname{sign}\left(\sigma^{(n-1)}\right)
$$

\footnotetext{
${ }^{1}$ To obtain the structure in (1) it is assumed that appropriate pre-transformations of the original full order dynamics and a (smooth) feedback control law have already been applied to 'cancel' known terms - in other words classical feedback linearization is used to obtain (1).
} 
where the scalars $\gamma_{1}, \gamma_{2}, \ldots, \gamma_{n}$ are such that the polynomial $p^{n}+\gamma_{n} p^{n-1}+\ldots+\gamma_{2} p+\gamma_{1}$ is Hurwitz and the scalars $\alpha_{1}, \alpha_{2}, \ldots, \alpha_{n}$ are chosen recursively as

$$
\alpha_{i-1}=\frac{\alpha_{i} \alpha_{i+1}}{2 \alpha_{i+1}-\alpha_{i}}, i=2, \ldots, n
$$

with $\alpha_{n+1}=1$ and $\alpha_{n}=\bar{\alpha}$. Then there exists an $\epsilon_{b} \in(0,1)$ such that for every $\bar{\alpha} \in\left(1-\epsilon_{b}, 1\right)$ the origin $\sigma, \dot{\sigma} \ldots, \sigma^{(n)}=0$ is a finite time stable equilibrium.

Proof: See Proposition 8.1 in [3].

This is clearly an existence theorem and the gains $\alpha_{i}$ must be computed by simulation. ${ }^{2}$ The table below shows an example of a set of gains which satisfies the conditions of Proposition 1 and achieves finite time convergence for systems of order up to 4 with the roots of the polynomial $p^{n}+\gamma_{n} p^{n-1}+\ldots+\gamma_{2} p+\gamma_{1}$ all equal to -2 .

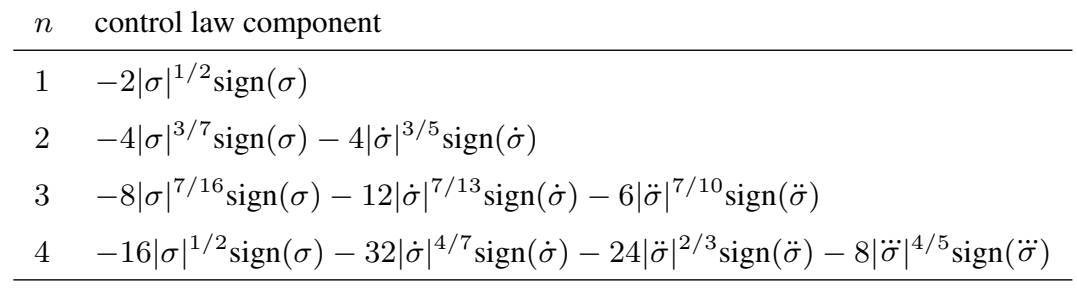

Table 1: Coefficients for the continuous finite time component

The second proposition considers the differential equations

$$
\begin{aligned}
& \dot{s}(t)=-\lambda(t) \operatorname{sign}(s(t))|s(t)|^{1 / 2}+z(t)-s(t) \dot{L}(t) / L(t) \\
& \dot{z}(t)=-\beta(t) \operatorname{sign}(s(t))+\phi(t)
\end{aligned}
$$

studied in [7]. In the above the variables $s, z$ are scalars and $\phi$ represents an unknown scalar uncertainty/disturbance which is differentiable with bounds which are unknown. In (4)-(5) the adaptive gains

$$
\begin{aligned}
\lambda(t) & =\sqrt{L(t)} \lambda_{0} \\
\beta(t) & =L(t) \beta_{0}
\end{aligned}
$$

\footnotetext{
${ }^{2}$ Recall the HOSMCs of [11] also rely on computer tuned gains.
} 
where $\lambda_{0}$ and $\beta_{0}>1$ are fixed positive scalars and $L(t)>0$ is an adjustable gain. Define three matrices as

$$
A_{0}=\left[\begin{array}{cc}
-\frac{1}{2} \lambda_{0} & \frac{1}{2} \\
-\beta_{0} & 0
\end{array}\right] \quad B_{0}=\left[\begin{array}{l}
0 \\
1
\end{array}\right] \quad C_{0}=\left[\begin{array}{ll}
1 & 0
\end{array}\right]
$$

then the following can be proved:

Proposition 2: (Edwards \& Shtessel) Suppose $L(t)$ is bounded and chosen to enforce $L(t) \geq|\phi(t)|$, then a 2-SM occurs making $s=\dot{s}=0$ in finite time if the gains $\lambda_{0}$ and $\beta_{0}$ are chosen so that there exists a symmetric positive definite matrix $P$ such that

$$
P A_{0}+A_{0}^{T} P+\tilde{\epsilon}_{0} P+P B_{0} B_{0}^{T} P+C_{0}^{T} C_{0}<0
$$

where the scalar $\tilde{\epsilon}_{0}>0$, or equivalently $\left\|G_{0}(s)\right\|_{\infty}<1$ where

$$
G_{0}(s):=C_{0}\left(s I-A_{0}\right)^{-1} B_{0}=\frac{1}{\left(2 s^{2}+\lambda_{0} s+\beta_{0}\right)}
$$

Proof: See Proposition 1 and Remark 2 in work of [7]

In Proposition 2 above the scalar $L(t)$ is considered as an adaptable gain which is 'advantageously' manipulated. Here the evolution of $L(t)$ will be chosen to depend on the so-called equivalent control. This theoretical abstraction proposed in [17] is usually used to analyze the reduced order dynamics associated with the sliding motion. During sliding, the equivalent control can be approximated in real-time by low pass filtering of the switched signal e.g

$$
\dot{\bar{u}}_{e q}(t)=\frac{1}{\tau}\left(\beta(t) \operatorname{sign}(s(t))-\bar{u}_{e q}(t)\right)
$$

where $\tau$ is a (small) positive constant. Furthermore, at least in principle, during sliding, the difference between $\bar{u}_{e q}(t)$ and the true value of the equivalent control $u_{e q}(t)$ can be made arbitrary small by making $\tau$ small [14]. Consequently, during the sliding motion exhibited in (4)-(5), by filtering the discontinuous injection signal $\beta(t) \operatorname{sign}(s(t))$, a good estimate of $u_{e q}(t)=\phi(t)$ (in this case) can be obtained in real-time provided $\tau$ is sufficiently small. In modern micro-processor boards with bespoke implementations of standard low pass filters, very high sample rates can be achieved allowing very small 
values of $\tau$ to be selected. For a detailed discussion on estimating the equivalent control see $[18,6]$.

Remark 1: The choice of the positive scalar $\tau$ is crucial to the performance of the controller. The scalar $\tau$ must be small enough so that the bandwidth of the filter in (11) is sufficiently high to capture all the important frequency components of $d(t)$ and yet be sufficiently practical so that unwanted noise does not become part of $\bar{u}_{e q}(t)$. In addition, taking into account possible digital implementation, $\tau$ should be larger than the sampling time of the chip on which the controller with the filter (11) is implemented. Invariably the choice of $\tau$ must be made on a case by case basis exploiting engineering system information/judgement concerning the plant to be controlled. The output of the filter in (11) is a visible signal during any simulation/implementation and so a judgement can be made from $\bar{u}_{e q}(t)$ whether or not a meaningful disturbance estimate for the plant is being obtained, and hence whether the selection of $\tau$ requires further tuning.

\section{Main Results}

Here, as in $[6,18]$ it is assumed a low-pass filter exists, with suitably small time constant $\tau$ and output $\bar{u}_{e q}(t)$, for which there exist scalars $0<\epsilon_{1}<1$ and $\epsilon_{0}>0$ such that

$$
|| \bar{u}_{e q}(t)|-| u_{e q}(t)||<\epsilon_{1}\left|u_{e q}(t)\right|+\epsilon_{0}
$$

holds for all time after a finite time $t_{e q}$. (The initial time interval $\left[0, t_{e q}\right]$ allows for the fast dissipation of the effects of the initial conditions in the filter (11).) For a given value of $\tau$, estimates of the scalars $\epsilon_{0}$ and $\epsilon_{1}$ can be computed to ensure (12) holds [6].

This paper proposes a new continuous adaptive control law with two varying parameters, specifically:

$$
u(t)=-u_{\sigma}(t)-u_{s}(t)
$$

where

$$
u_{\sigma}(\cdot)=\gamma_{1}|\sigma|^{\alpha_{1}} \operatorname{sign}(\sigma) \ldots+\gamma_{n}\left|\sigma^{(n-1)}\right|^{\alpha_{n}} \operatorname{sign}\left(\sigma^{(n-1)}\right)
$$


(which is identical to the right hand side of (2)) and

$$
u_{s}(t)=s(t) \dot{L}(t) / L(t)+\lambda(t)|s|^{1 / 2} \operatorname{sign}(s)+\int_{0}^{t} \beta(\tau) \operatorname{sign}(s(\tau)) d \tau
$$

(which is new) where the auxiliary sliding variable $s$ is defined as

$$
s(t)=\sigma^{(n-1)}(t)+\int_{0}^{t} u_{s}(\tau) d \tau
$$

In (15) the time varying gain

$$
\lambda(t)=2 \sqrt{2 \beta_{0} L(t)}
$$

and $\beta(t)$ is defined in (7), where $\beta_{0}>1$ is a fixed design scalar and $L(t)>l_{0}>0$ is a time-varying gain. In (14) the scalars $\gamma_{1}, \gamma_{2}, \ldots, \gamma_{n}$ must be chosen so that the polynomial $p^{n}+\gamma_{n} p^{n-1}+\ldots+\gamma_{2} p+\gamma_{1}$ is Hurwitz and the scalars $\alpha_{1}, \alpha_{2}, \ldots, \alpha_{n}$ are chosen recursively to satisfy the conditions in (3) associated with Proposition 1.

Theorem 1: Consider the system in (1) with uncertainty $d(t)$ which is twice differentiable and subject to $|\dot{d}(t)|<d_{1}$ and $|\ddot{d}(t)|<d_{2}$ where the bounded scalars $d_{1}$ and $d_{2}$ exist but are unknown. Using the control law from (13) in (1) where $L(t)$ is adapted in such a way that $L(t)>|\dot{d}(t)|$, then there exists an $\epsilon_{b} \in(0,1)$ such that for every $\bar{\alpha} \in\left(1-\epsilon_{b}, 1\right)$ the origin $\sigma, \dot{\sigma}, \ldots, \sigma^{(n)}=0$ is a finite time stable equilibrium point.

Proof: From equations (16), (1), (13) and (15), the dynamics associated with the auxiliary variable $s$ are described by

$$
\dot{s}(t)=d(t) \underbrace{-\lambda(t)|s|^{1 / 2} \operatorname{sign}(s)-\int_{0}^{t} \beta(\tau) \operatorname{sign}(s(\tau)) d \tau-\dot{L}(t) s(t) / L(t)}_{-u_{s}(t)}
$$

Define $\phi(t)=\dot{d}(t)$, then (18) is exactly equivalent to the set of differential equations in (4)-(5) studied in [7]. Using $\lambda_{0}=2 \sqrt{2 \beta_{0}}$ where $\beta_{0}>1$, it is easy to confirm from (10) that $\left\|G_{0}(s)\right\|_{\infty}<1$ and using Proposition 2 from Section 3 the choice of gains in (7) and (17) ensure a 2-SM occurs in finite time in (4)-(5) if $L(t)>|\phi(t)|$, forcing $s=\dot{s}=0$. Once sliding has been attained and $\dot{s}=s=0$, the term $u_{s}(t)$ from (13) exactly compensates for the uncertainty: i.e $u_{s}(t)=d(t)$. Consequently from equations (1) and (13)

$$
\sigma^{(n)}=d(t)+u(t)=\underbrace{d(t)-u(t)}_{=0}-u_{b}(t)=-u_{b}(t)
$$


and therefore from the definition of $u_{b}$ in (14), during the sliding motion (associated with $s$ ) the closed-loop system is governed by

$$
\sigma^{(n)}=-\gamma_{1}|\sigma|^{\alpha_{1}} \operatorname{sign}(\sigma) \ldots-\gamma_{n}\left|\sigma^{(n-1)}\right|^{\alpha_{n}} \operatorname{sign}\left(\sigma^{(n-1)}\right)
$$

From Proposition 1 in Section 3, there exists an $\epsilon_{b} \in(0,1)$ such that for every $\bar{\alpha} \in$ $\left(1-\epsilon_{b}, 1\right)$ the origin $\sigma, \dot{\sigma} \ldots, \sigma^{(n)}=0$ is a finite time stable equilibrium

Based on the result in Theorem 1, the problem becomes one of selecting $L(t)$ so that $L(t)>|\phi(t)|$. In this paper this constraint will be ensured by using the dual-layer adaptive structure proposed in [6]. As in [6] define a new scalar variable $\delta$ as

$$
\delta(t)=L(t)-\frac{1}{\alpha \beta_{0}}\left|\bar{u}_{e q}(t)\right|-\epsilon
$$

In (19), $\bar{u}_{e q}(t)$ is the approximation of the equivalent control, the scalar $\alpha$ is chosen to satisfy $0<\alpha<1 / \beta_{0}<1$ and $\epsilon$ is a small positive scalar chosen to ensure

$$
\frac{1}{\alpha \beta_{0}}\left|\bar{u}_{e q}(t)\right|+\epsilon / 2>\frac{1}{\beta_{0}}\left|u_{e q}(t)\right|
$$

The design scalars $\alpha$ and $\epsilon$ represent 'safety margins' (at the expense of introducing conservatism) and are discussed in Remark 3.

The proposed adaptive element $L(t)$ on which the gains $\lambda(t)$ and $\beta(t)$ depend is given by

$$
L(t)=l_{0}+l(t)
$$

where $l_{0}$ is a (small) fixed positive (design) constant and

$$
\dot{l}(t)=-\rho(t) \operatorname{sign}(\delta(t))
$$

where $\delta(t)$ is defined in (19). The scalar in (22) is by definition

$$
\rho(t)=r_{0}+r(t)
$$

where $r_{0}$ is a fixed positive design scalar and the time varying component $r(t)$ satisfies

$$
\dot{r}(t)=\gamma|\delta(t)|
$$

where $\gamma$ is a fixed positive scalar design constant. 
Remark 2: It is clear the adaption scheme is driven by the estimate of the equivalent control $\bar{u}_{e q}(t)$. The estimate of the equivalent control $\bar{u}_{e q}(t)$ is typically (almost always) obtained by low-pass filtering the discontinuous injection. The choice of bandwidth of the filter (which constitutes design freedom) affects the accuracy with which $\bar{u}_{e q}(t)$ captures the true formal equivalent control and hence the disturbance/uncertainty. In the scheme proposed in this paper the signal $\bar{u}_{e q}(t)$ does not need to perfectly capture the equivalent control but needs to be sufficiently accurate to satisfy (12). To select an appropriate bandwidth, engineering understanding of the physical system to be controlled is required - especially an understanding of the relevant frequency ranges of the disturbances. The choice of filter in this sense plays a similar role to the weighting functions used in robust linear control when formulating $\mathcal{H}_{\infty}$ problems [19].

Theorem 2: The controller given in (13)-(17) and (21)-(24) is a continuous adaptive HOSM controller for the system in (1), and drives $\sigma, \dot{\sigma}, \ldots, \sigma^{(n)} \rightarrow 0$ in finite time despite the presence of the disturbance $d(t)$ with bounded derivatives $|\dot{d}(t)| \leq d_{1}$ and $|\ddot{d}(t)|<d_{2}$ without requiring knowledge of $d_{1}$ and $d_{2}$.

Proof: As shown in the proof of Proposition 2 in [7] the dual-layer adaptive mechanism in (21)-(24) forces $L(t)>|\phi(t)|$ in finite time. Once achieved, the conditions of Theorem 1 above are satisfied and $\sigma, \dot{\sigma}, \ldots, \sigma^{(n)}=0$ in finite time and the statement of the theorem is proven.

Remark 3: In [7] it is argued that for the scheme to work successfully, the true equivalent control $u_{e q}(t)$ must satisfy

$$
\frac{1}{\beta_{0}}\left|u_{e q}(t)\right| \leq \frac{1}{\beta_{0}}\left|\bar{u}_{e q}(t)\right|+\underbrace{\frac{1-\alpha}{\alpha \beta_{0}}\left|\bar{u}_{e q}(t)\right|+\frac{\epsilon}{2}}_{\text {safety margin }}
$$

In the right hand side of (25) it is clear the gains $\alpha$ and $\epsilon$ establish a 'cone' around the equivalent control $\left|u_{e q}(t)\right|$ involving the multiplicative gain $\frac{(1-\alpha)}{\alpha \beta_{0}}$ and a fixed off-set $\frac{\epsilon}{2}$. These introduce a safety margin and robustness into the adaptive scheme - which is crucial since $\left|u_{e q}(t)\right|$ can only be estimated by $\bar{u}_{e q}(t)$ through the low-pass filtering process. A small value of $\alpha \ll 1$ and a large value for $\epsilon$ reflects a lack of confidence in $\bar{u}_{e q}(t)$ capturing accurately the true value of the equivalent control and therefore 
the actual uncertainty. A consequence of using a small value of $\alpha \ll 1$ and a large value for $\epsilon$ is that the gain $L(t)$ in (19) no longer accurately tracks the magnitude of the uncertainty (since it tracks the right hand side of (25)) and therefore the adaptive gains will be unnecessarily large. Conversely a value of $\alpha$ close to unity and a small value for $\epsilon$ means $L(t)$ will closely track the magnitude of the uncertainty; however to achieve this requires confidence in $\bar{u}_{e q}(t)$ accurately reflecting the true equivalent control. (If $\alpha=1$ and $\epsilon=0$ then $\left|u_{e q}(t)\right|=\left|\bar{u}_{e q}(t)\right|$ must hold.)

Remark 4: Prior to sliding taking place, filtering the injection term $\beta(t) \operatorname{sign}(\sigma)$ does not formally provide 'the equivalent injection' (which only has meaning during the existence of a sliding motion). However in the absence of sliding it can be shown that $\operatorname{sign}(\delta(t))<0$ and therefore $i(t)>r_{0}>0$ and the gain $L(t)$ is forced to monotonically increase. Once $L(t)$ is sufficiently large, sliding is induced in finite time. For further details see [6].

Remark 5: Compared to the control law described in [6] the significant difference to the scheme presented in this paper is the definition of $u_{s}(t)$ in $(15)$ and the specific choice of the adaptive gains in (7) and (17) which allow both $\lambda(t)$ and $\beta(t)$ to adapt. Previously the scheme in [6] involved a fixed 'large enough $\lambda$ '. By allowing $\lambda(t)$ to vanish as $L(t)$ becomes small helps to mitigate chattering.

Remark 6: For practical implementation the adaptive scheme in (22) and (24) can be replaced by

$$
\begin{aligned}
& \dot{l}(t)=-\rho(t) \operatorname{sign}(\delta(t)) \\
& \dot{r}(t)= \begin{cases}\gamma|\delta(t)| & \text { if }|\delta(t)|>\delta_{0} \\
0 & \text { otherwise }\end{cases}
\end{aligned}
$$

where $\delta_{0}$ is a (small) positive design scalar. For details see [6].

Remark 7: In both versions of the adaptation law (i.e (24) and (27)) the gain $r(t)$ and hence $\rho(t)$ is non-decreasing but remains bounded. The gain $\rho(t)$ can be interpreted as an upper bound on the second derivative of the disturbance $|\ddot{d}(t)|$. Although in (22) and (26) large amplitude switching can occur in the right hand side if $\rho(t)$ is large, the gain $l(t)$, and hence $L(t)$, are both continuous. Consequently the changes to $\lambda(t)$ and $\beta(t)$ in (6) and (7) are continuous. 


\section{Simulations}

Consider the relative degree three situation when $n=3$ in (1) and where for simulation purposes the unknown disturbance is given by $d(t)=2 \sin t+0.2 \sin 5 t+0.5$. The signal $d(t)$ and the bounds on the derivatives $\dot{d}(t)$ and $\ddot{d}(t)$ are assumed to be unknown. In the simulations, the values from Table 1 have been used to create $u_{\sigma}(t)$ in (14). In the adaptive component $\beta_{0}=1.1, \gamma=10, r_{0}=1$ and $l_{0}=0.001$. The time constant of the filter (11) is $\tau=0.001$, starting with zero initial conditions. The results of the simulations, obtained by the Euler integration algorithm with a fixed time increment equal to $10^{-6} s$, where $\sigma(0)=1, \dot{\sigma}(0)=0.5$ and $\ddot{\sigma}(0)=0$, in three different scenarios are shown in the sequel. The three simulation scenarios considered here are:

1. With the HOSM controller parameters $\alpha=0.99$ and $\epsilon=0.02$ in (19).

2. With the HOSM controller parameters $\alpha=0.7$ and $\epsilon=0.3$ in (19). This case deliberately introduces larger safety margins in the control gain $\beta(t)$ and results in conservative over-bounding of the unknown the perturbation derivative $|\dot{d}|$.

3. Using the continuous adaptive 3-SMC with $\lambda(t)=\lambda(0)=0.05$ (i.e. the scheme from [5] in which $\lambda$ is non-adaptive) and only $\beta(t)$ is adapted in accordance with equations (7) and (21)-(24))

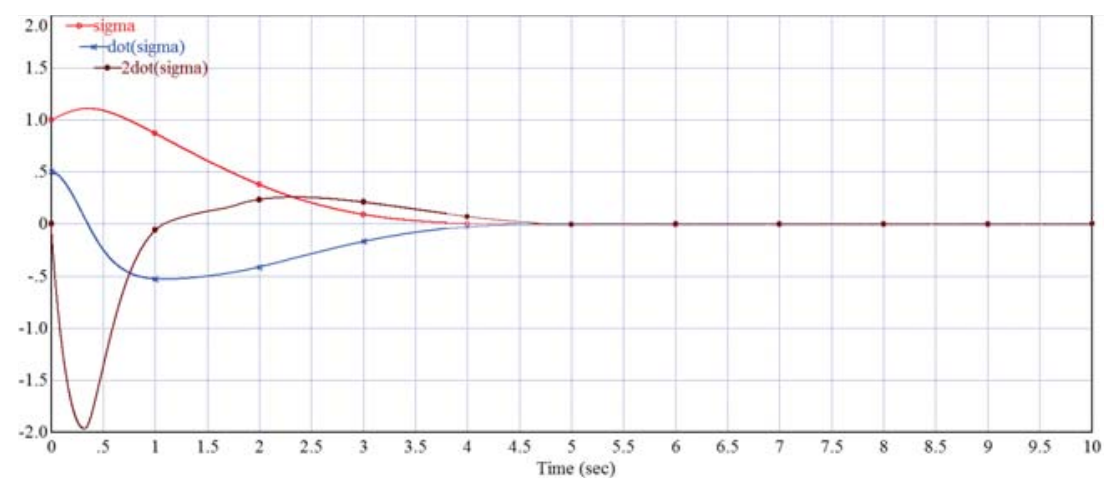

Figure 1: Evolution of $\sigma, \dot{\sigma}$ and $\ddot{\sigma}$ with the adaptive gains $\lambda(t)$ and $\beta(t)$

The plots for the first simulation scenario are shown in Figures 1-4. Figure 1 demonstrates finite time convergence of $\sigma, \dot{\sigma}$ and $\ddot{\sigma}$ to zero in the presence of the bounded 


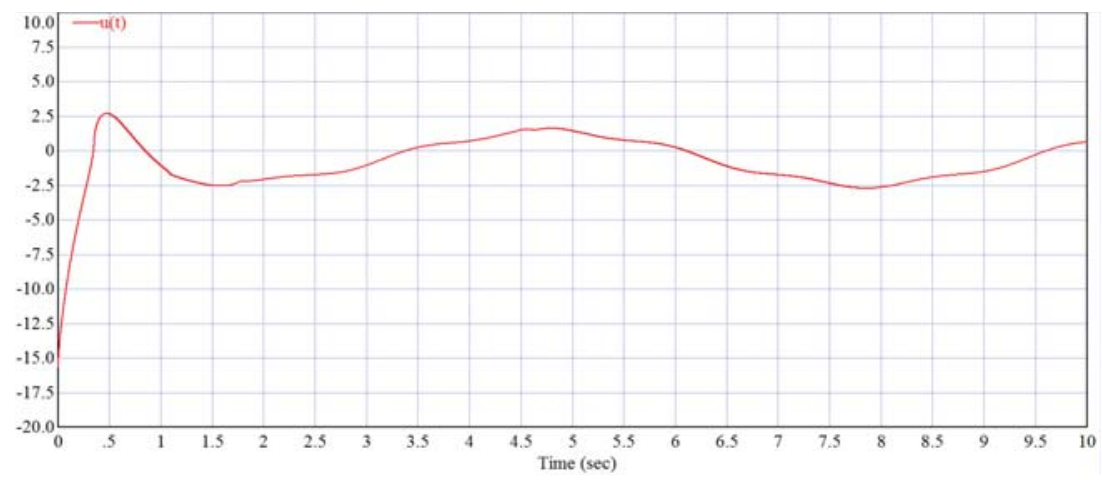

Figure 2: Evolution of the control function $u(t)$

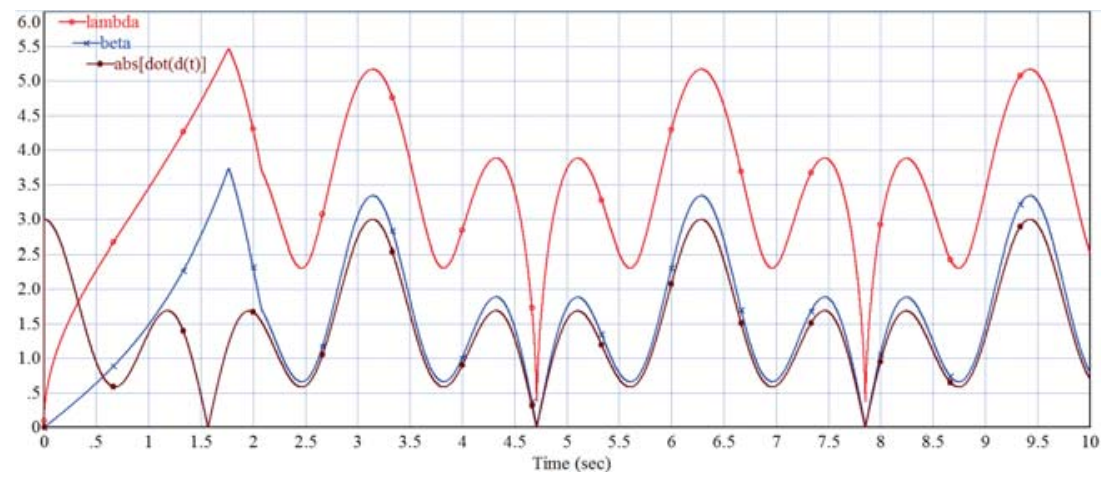

, .

Figure 3: Evolution of the adaptive gains $\lambda(t)$ and $\beta(t)$ and $|\dot{d}(t)|$ for $\alpha=0.99$ and $\epsilon=0.02$

disturbance. The time history of the continuous adaptive 3-SMC control $u(t)$, with both $\lambda(t)$ and $\beta(t)$ being adapted, is shown in Figure 2. The evolution of the gains $\lambda(t)$ and $\beta(t)$, shown in Figure 3, demonstrates the excellent self-tuning capabilities of the adaptive continuous 3-SMC control from (6)-(7), (21)-(24) in terms of control gain non-overestimation. Specifically, the adaptive gain $\beta(t)$ just slightly supersedes the $|\dot{d}(t)|$ profile, while $\lambda(t)=2 \sqrt{2 \beta(t)}$ is in accordance with equations (7) and (17).

The evolutions of $\rho(t), \delta(t)$ and $L(t)$ are presented in Figure 4. It is clear that the adaptive gain $\rho(t)$ reaches a constant value, while the adaptive parameter $L(t)$ follows 


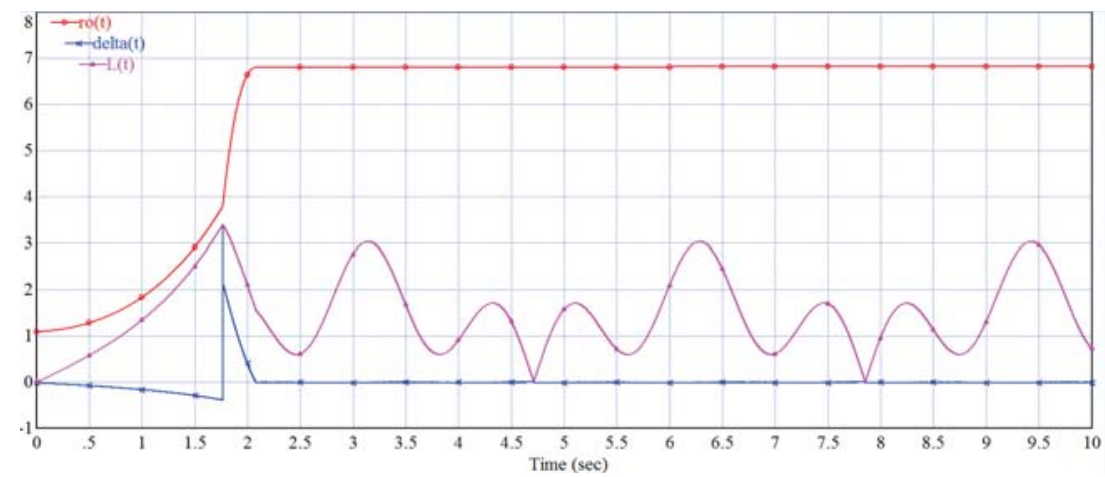

Figure 4: Evolution of $\rho(t), \delta(t)$ and $L(t)$ for $\alpha=0.99$ and $\epsilon=0.02$

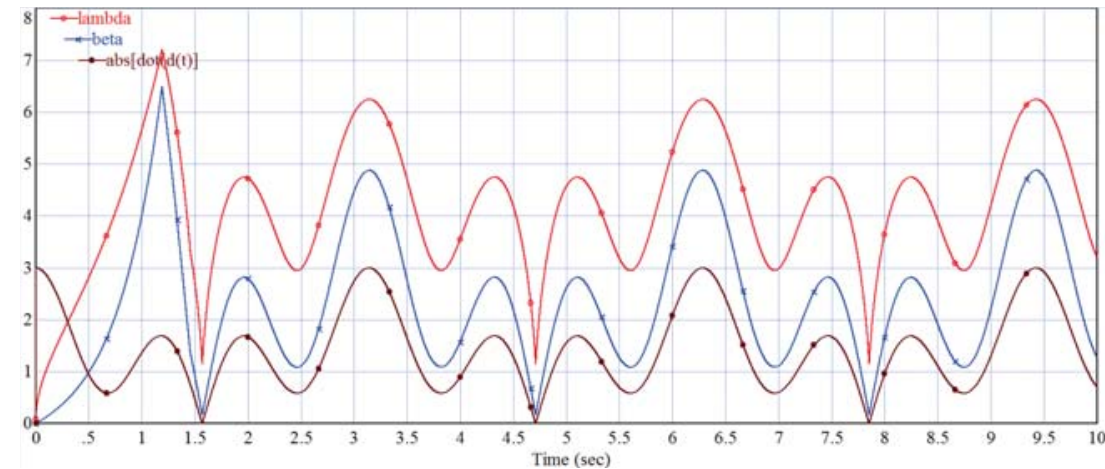

Figure 5: Evolution of $\lambda(t), \beta(t)$ and $|\dot{d}(t)|$ for $\alpha=0.7$ and $\epsilon=0.3$ 
the unknown profile $|\dot{d}(t)|$, and the variable $\delta(t)$ reaches zero in finite time.

The plots for the second simulation scenario are shown in Figure 5 and demonstrates the adaptive gain $\beta(t)$ over-bounds the profile of $|\dot{d}(t)|$ with a larger more conservative margin than in the first simulation scenario: this is exactly as expected. The plots of $\sigma, \dot{\sigma}$, and $\ddot{\sigma}$ as well of the control function $u(t)$ are practically the same as in the first scenario.

The plots for the third simulation scenario are shown in Figures 6 and 7 and demonstrate a significant degradation of the adaptive control system performance with a fixed (poorly) chosen value of $\lambda(t)=0.05$. Specifically, the transient responses of $\sigma, \dot{\sigma}$ and $\ddot{\sigma}$ in Figure 6 are much more oscillatory, and the control function $\mathrm{u}(\mathrm{t})$ in Figure 7 is no longer continuous.

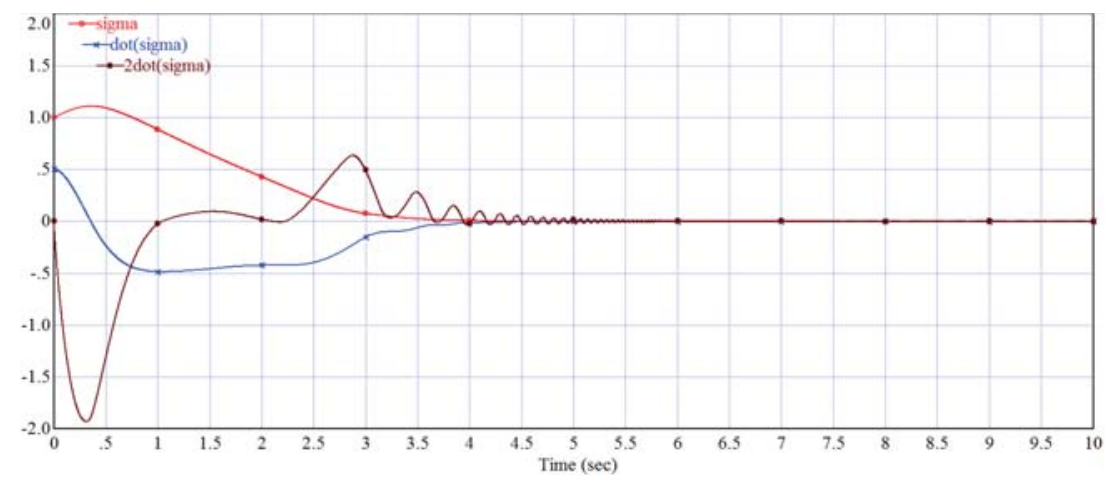

Figure 6: Evolution of $\sigma, \dot{\sigma}$ and $\ddot{\sigma}$ with the adaptive gain $\beta(t)$ and fixed $\lambda=0.05$

\section{Conclusions}

The main contribution of this paper is in proposing a Continuous Adaptive HOSM control algorithm. The adaptive scheme does not require knowledge of upper bounds on the (matched) uncertainty and its derivatives, and is constructed so as not to conservatively overestimate the magnitude of the uncertainty, thus mitigating chattering. Compared with the authors' earlier work, in this paper, two parameters adapt and this allows better self-tuning. 


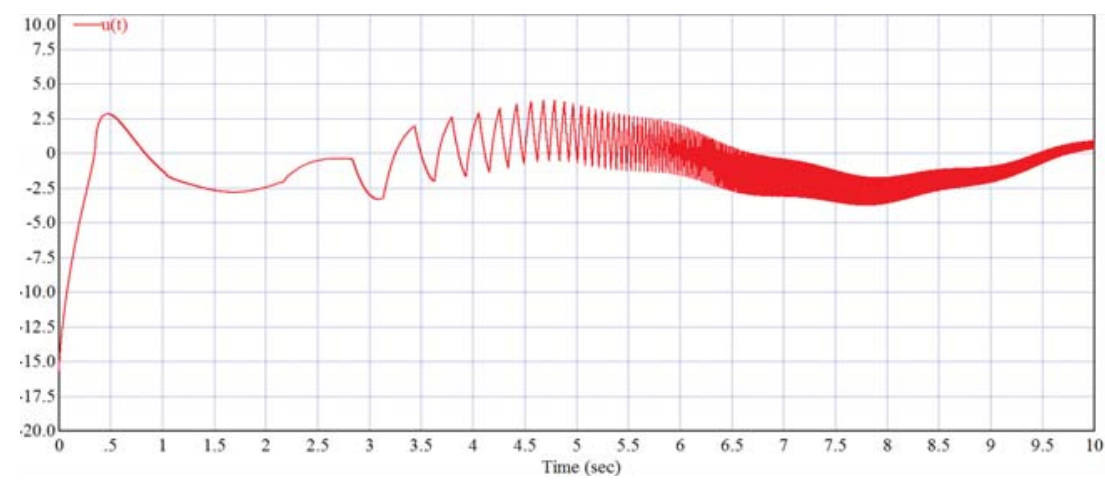

Figure 7: Evolution of $u(t)$ with the adaptive gain $\beta(t)$ and fixed $\lambda=0.05$

\section{References}

[1] H. Alwi and C. Edwards. An adaptive sliding mode differentiator for actuator oscillatory failure case reconstruction. Automatica, 49:642-651, 2013.

[2] M.V. Basin, C.B. Panathula, and Y. Shtessel. Adaptive uniform finite-/fixedtime convergent second-order control, International Journal of Control, 89:17771787, 2016.

[3] S. Bhat and D.S. Bernstein. Geometric homogeneity with applications to finitetime stability. Math. Control Signals Systems, 17:101-127, 2005.

[4] I. Boiko. Discontinuous Control Systems: Frequency-Domain Analysis and Design. Birkhauser, 2009.

[5] C. Edwards and S. K. Spurgeon. Sliding Mode Control: Theory and Applications. Taylor \& Francis, 1998.

[6] C. Edwards, and Y. Shtessel. Adaptive continuous higher order sliding mode control, Automatica, 65, 183-190, 2016.

[7] C. Edwards and Y. Shtessel. Adaptive Dual Layer Super-Twisting Control and Observation International Journal of Control, 89, 1759-1766, 2016. 
[8] A.F. Filippov. Differential equations with discontinuous right hand-sides. American Mathematical Society Translations, 42:199-231, 1964.

[9] L. Fridman and A. Levant. Higher order sliding modes. In W. Perruquetti and J.P. Barbot, editors, Sliding Mode Control in Engineering, pages 53-96. Marcel Dekker, New York, 2002.

[10] Y.-J. Huang, T.-C. Kuo, and S.-H. Chang. Adaptive sliding-mode control for nonlinear systems with uncertain parameters. IEEE Transactions on Systems, Man, and Cybernetics, Part B: Cybernetics, 38:534 - 539, 2008.

[11] A. Levant. Higher order sliding modes, differentiation and output feedback control. Interantional Journal of Control, 76:924-941, 2003.

[12] A. Levant. Quasi-continuous higher order sliding mode controllers. IEEE Transactions on Automatic Control, 50:1812-1816, 2006.

[13] F. Plestan, Y. Shtessel, V. Bregeault, and A. Poznyak. New methodologies for adaptive sliding mode control. International Journal of Control, 83:1907-1919, 2010.

[14] Y. Shtessel, C. Edwards, L. Fridman, and A. Levant. Sliding Mode Control and Observation. Birkhauser, 2013.

[15] Y. Shtessel, M. Taleb, and F. Plestan. A novel adaptive-gain supertwisting sliding mode controller: Methodology and application. Automatica, 48:759769, 2012.

[16] M. Taleb, A. Levant, and F. Plestan. Electropneumatic actuator control: solutions based on adaptive twisting algorithm and experimentation. Control Engineering Practice, 21:727-736, 2013.

[17] V. I. Utkin. Sliding Modes in Control Optimization. Springer-Verlag, Berlin, 1992.

[18] V.I. Utkin and A.S. Poznyak. Adaptive sliding mode control with application to super-twisting algorithm: Equivalent control method. Automatica, 49:39-47, 2013. 
[19] S. Skogestad and I. Postlethwaite". Multivariable Feedback Control: Analysis and Design J.Wiley", 1996. 